\title{
LA SALIDA DE UN ESTADO MIEMBRO EN EL PROCESO DE INTEGRACIÓN. EL REINO UNIDO Y LA UNIÓN EUROPEA
}

\author{
A SAIIDA DE UM ESTADO-MEMBRO NO PROCESSO DE \\ INTEGRAÇÃO. O REINO UNIDO E A UNIÃO EUROPEIA
}

Roberto Ruiz Díaz Labrano*

Resumen: La integración europea, más allá de sus dificultades, ha sido y sigue siendo un ejemplo de un proceso de integración exitoso, ha tenido un desarrollo extraordinario y una expansión siempre creciente de adhesión de Estados que se han incorporado al bloque.

Conforme a sus objetivos el esquema fue afianzándose y aun cuando ha debido transitar crisis y diversas situaciones difíciles, por primera vez se presenta una situación en la cual uno de sus Estados Miembros, el Reino Unido, ubicado entre los tres más destacados en el aporte presupuestario, decide retirarse de la Unión Europea como resultado de un referéndum. El hecho golpea fuertemente a la cohesión que en su conjunto siempre ha demostrado la Unión y requerirá de un profundo análisis sobre los pasos futuros a fin de evitar situaciones similares o un efecto contagio, sobre todo por sectores nacionalistas que pueden encontrar en el planteo del "exit" encender el debate político.

La decisión tiene serias consecuencias para el Estado saliente, el Reino Unido tendrá que utilizar una enorme creatividad y poder de negociación para reubicarse en el nuevo contexto para sus negociaciones internacionales. El presente artículo se busca explicar de algún modo lo ocurrido y las consecuencias de la decisión, así como el mecanismo de negociación a partir de la comunicación de la decisión.

Resumo: A integração europeia, ainda de suas dificuldades, foi e segue sendo um exemplo de processo de integração exitoso. Ela teve um desenvolvimento extraordinário e uma expansão sempre crescente de adesão de Estados que se incorporaram ao bloco.

\footnotetext{
* Doctor en Derecho. Miembro Titular del Tribunal Permanente de Revisión del Mercosur. Profesor Titular de Derecho Internacional Privado de la Facultad de Derecho de la Universidad Nacional de Asunción. Profesor Titular de Derecho de la Integración de la Facultad de Derecho de la Universidad Nacional de Asunción. Profesor de la Maestría en Derecho Internacional Privado y de la Integración de la Universidad de Buenos Aires. Miembro honorarios de la Asociación Americana de Derecho Internacional Privado “ASADIP”. E-mail: rlabrano@rdlabrano.com
} 
Conforme aos seus objetivos o esquema foi-se afiançando e ainda quando teve que transitar crises e diversas situações difíceis, pela primeira vez, se apresenta uma situação na qual um de seus Estados Membros, o Reino Unido, que fica entre os três Estados más destacados no aporte orçamentário, decide se retirar da União Europeia, como resultado de um referendum.

O fato golpeia fortemente à coesão que no seu conjunto sempre demonstrou a União e requererá de uma aprofundada analise sobre os passos futuros aos fins de evitar situações similares o um efeito contagio, sobre todo pelos setores nacionalistas que podem encontrar no "exit" o início do debate político.

A decisão tem sérias consequências para o Estado que fica saindo, o Reino Unido terá que utilizar uma grande criatividade e poder de negociação para ficar de volta no novo contexto para suas negociações internacionais. No presente artigo procura-se explicar, de algum jeito, o acontecido e as consequências da decisão, assim como o mecanismo de negociação a partir da comunicação da decisão.

Palabras clave: Unión Europea, Pertenencia a la Unión Europea, Reino Unido, Brexit

Palavras-chave: União Europeia, Pertença na União Europeia, Reino Unido, Brexit

\section{INTRODUCCIÓN}

La integración supone un acuerdo de voluntades estatales, el deseo de ser parte de una meta común con proyectos y objetivos comunes, para tal efecto los Estados asumen una serie de responsabilidades obligaciones - y se benefician de derechos y beneficios comunes como resultado de la Unión.

Los resultados de la integración deben beneficiar a todos los Estados miembros, no sólo a algunos, debe producir esa sensación en los ciudadanos para que éstos sigan acompañando el proceso, situación fácil de apreciar cuando los protagonistas son pocos, pero muy difícil cuando aumenta la cantidad de miembros con dimensiones, necesidades, ambiciones y realidades diferentes.

En tal sentido y para analizar lo que condujo al Reino Unido a un referéndum que voto por el sí para la salida de la Unión Europea, hemos considerado necesario recordar primero cuales han sido los grandes delineamientos y objetivos fundamentales que fueran propuestos en su momento para el proceso de integración europeo.

Por el tratado constitutivo de 1992 las altas partes contratantes crean entre sí la Unión Europea y como elemento principal la unidad 
respecto a un objetivo político geográfico la construcción y consolidación de Europa como un todo. Se deja en claro que es un "proceso creador" de unidad buscando estrechar lazos entre los pueblos de Europa, aproximando las decisiones a los ciudadanos.

Se propuso en aquel momento que la Unión “Tendrá por misión organizar de modo coherente y solidario las relaciones entre los Estados

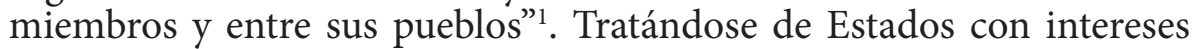
diversos, la armonización de los intereses ha sido eje para la organización de las relaciones entre los miembros y los ciudadanos de cada uno de ellos, estableciéndose indicativamente las formas de cooperación.

Se podrá notar que las expresiones, solidaridad, cooperación, coherencia para la organización de las relaciones de los Estados miembros, fue el motor fundamental para lograr apuntalar un "proceso creador" capaz de lograr el objetivo de alcanzar la Unión Europea o la unión de todos los Estados Europeos.

Entre los principales objetivos, se acordó promover en un espacio sin fronteras "un progreso económico y social equilibrado y sostenible". Se propuso lograr el "fortalecimiento de la cohesión económica y social y el establecimiento de una unión económica y monetaria”. Ya en aquél momento apuntando a la moneda única. Otros objetivos, como la afirmación de la entidad internacional, la seguridad común encaminada a otro gran objetivo el de la "defensa común".

Se dispuso tener la suficiente flexibilidad en el ritmo de integración, a fin de garantizar que las políticas de cooperación fueran revisadas o reajustadas y teniendo presente el principio de subsidiariedad. Como otro gran objetivo se apuntó a la ciudadanía común europea ${ }^{2}$. Con el

1 TRATADO DE LA UNIÓN EUROPEA (92/C 191/01). TÍTULO I. DiSPOSICIONES COMUNES. Artículo A. Por el presente Tratado, las Altas Partes Contratantes constituyen entre sí una Unión Europea, en lo sucesivo denominada "Unión". El presente Tratado constituye una nueva etapa en el proceso creador de una Unión cada vez más estrecha entre los pueblos de Europa, en la cual las decisiones serán tomadas de la forma más próxima posible a los ciudadanos. La Unión tiene su fundamento en las Comunidades Europeas completadas con las políticas y formas de cooperación establecidas por el presente Tratado. Tendrá por misión organizar de modo coherente y solidario las relaciones entre los Estados miembros y entre sus pueblos.

2 TRATADO DE LA UNIÓN EUROPEA (92/C 191/01). Artículo B. La Unión tendrá los siguientes objetivos: - promover un progreso económico y social equilibrado y sostenible, principalmente mediante la creación de un espacio sin fronteras interiores, el fortalecimiento de la cohesión económica y social y el establecimiento de una unión económica y monetaria que implicará, en su momento, una moneda única, conforme a las disposiciones del presente Tratado, - afirmar su identidad en el ámbito internacional, en particular mediante la realización de una política exterior y de seguridad común que incluya, en el futuro, la definición de una política de defensa común que podría conducir, en su momento, a una defensa común, reforzar la protección de los derechos e intereses de los nacionales de sus Estados miembros, mediante la creación de una ciudadanía de la Unión, - desarrollar una cooperación estrecha en el ámbito de la justicia y de los asuntos de interior, - mantener íntegramente el acervo comunitario y desarrollarlo con el fin de examinar, con arreglo al procedimiento previsto en el apartado 2 del artículo $\mathrm{N}$, la medida en que las políticas y formas de cooperación establecidas en el presente Tratado deben ser revisadas, para asegurar la eficacia de los 
Tratado de Ámsterdam se apuntó a lograr "un alto nivel de empleo"3.

Se encomendó que con en el marco institucional único creado, se buscará la coherencia y la continuidad de las acciones para alcanzar los objetivos, esencialmente velando por "mantener la coherencia del conjunto de su acción exterior en el marco de sus políticas en materia de relaciones exteriores, de seguridad, de economía y de desarrollo", encomendando al Consejo y a la Comisión la responsabilidad de garantizar la coherencia ${ }^{4}$.

Se dispuso además y de forma expresa que "La Unión respetará la identidad nacional de sus Estados miembros, cuyos sistemas de gobierno se basarán en los principios democráticos" 5 . Con el Tratado de la Unión se acordó "el establecimiento de un mercado común y de una unión económica y monetaria”, mediante la realización de las políticas y acciones comunes.

En el mismo instrumento se apuntó a "un desarrollo armonioso y equilibrado de las actividades económicas en el conjunto de la Comunidad, un crecimiento sostenible y no inflacionista que respete el medio ambiente, un alto grado de convergencia de los resultados económicos, un alto nivel de empleo y de protección social, la elevación del nivel y de la calidad de vida, la cohesión económica y social y la solidaridad entre los Estados miembros" 6 .

Entendemos que bajo esta perspectiva y retrospectivamente debe analizarse la particular situación que se ha producido en la Unión Europea, atendiendo a la diversidad de intereses en juego frente a los

mecanismos e instituciones comunitarios. Los objetivos de la Unión se alcanzarán conforme a las disposiciones del presente Tratado, en las condiciones y según los ritmos previstos y en el respeto del principio de subsidiariedad tal y como se define en el artículo 3B del Tratado constitutivo de la Comunidad Europea.

3 Tratado de Ámsterdam, nuevo "Artículo B La Unión tendrá los siguientes objetivos: promover el progreso económico y social, un alto nivel de empleo y conseguir un desarrollo equilibrado y sostenible, principalmente mediante la creación de un espacio sin fronteras interiores, el fortalecimiento de la cohesión económica y social y el establecimiento de una unión económica y monetaria que implicará, en su momento, una moneda única, conforme a las disposiciones del presente Tratado".

4 TRATADO DE LA UNIÓN EUROPEA (92/C 191/01). Artículo C La Unión tendrá un marco institucional único que garantizará la coherencia y la continuidad de las acciones llevadas a cabo para alcanzar sus objetivos, dentro del respeto y del desarrollo del acervo comunitario. La Unión velará, en particular, por mantener la coherencia del conjunto de su acción exterior en el marco de sus políticas en materia de relaciones exteriores, de seguridad, de economía y de desarrollo. El Consejo y la Comisión tendrán la responsabilidad de garantizar dicha coherencia y asegurarán, cada cual conforme a sus competencias, la realización de tales políticas.

5 Artículo F. 1. La Unión respetará la identidad nacional de sus Estados miembros, cuyos sistemas de gobierno se basarán en los principios democráticos.

6 Artículo 2 La Comunidad tendrá por misión promover, mediante el establecimiento de un mercado común y de una unión económica y monetaria y mediante la realización de las políticas o acciones comunes contempladas en los artículos 3 y $3 \mathrm{~A}$, un desarrollo armonioso y equilibrado de las actividades económicas en el conjunto de la Comunidad, un crecimiento sostenible y no inflacionista que respete el medio ambiente, un alto grado de convergencia de los resultados económicos, un alto nivel de empleo y de protección social, la elevación del nivel y de la calidad de vida, la cohesión económica y social y la solidaridad entre los Estados miembros". 
principales objetivos que se propuso alcanzar con la integración.

\section{EL BREXIT}

En cuanto al Brexit ${ }^{7}$, hemos asistido sorprendidos al suceso que ha conmovido a la Unión Europea. Se convirtió de inmediato en noticia mundial por las repercusiones inmediatas y las que es razonable esperar. De inicio se puede decir que lo impensable, lo no deseable, lo insólito o lo absurdo ha ocurrido, el voto favorable al referéndum sobre la salida del Reino Unido.

Lo que parecía una consulta con respuesta preanunciada, el rechazo del sí a la salida del Reino Unido y que debía continuar siendo miembro de la Unión Europea, no fue tal, los ciudadanos británicos por escaso margen votaron por el retiro ${ }^{8}$.

De este modo y por primera vez en el proceso de integración europeo, un Estado Miembro luego de 43 años de haberse adherido, decide vía referéndum abandonar años de esfuerzos para unir y construir la integración Europa.

La lectura de este acontecimiento histórico en la vida del proceso de integración y que ocurre en el bloque actual más exitoso y más avanzado, tiene múltiples repercusiones internas e internacionales, económicas, políticas y sociales.

Como situación jurídica, recuerda que un componente esencial para la integración es la satisfacción de todos los Estados que lo conforman, porque es el elemento fundamental de cohesión para trazar un destino común. La insatisfacción o la sensación de insatisfacción pueden llevar a algunos pocos protagonistas, como ha ocurrido, asumir posiciones personales radicales y dañar profundamente los esfuerzos en pos de la integración, sin analizar en profundidad si el precio a pagar resulta alto.

El referéndum surge de una promesa electoral de David Cameron a su reelección como Primer Ministro en el 2015, él había prometido celebrar un referendo sobre la permanencia o no del Reino Unido en la Unión Europea.

La idea de restablecer popularidad cumpliendo su promesa y la certeza de que las negociaciones llevadas a cabo con Bruselas, que consideró suficiente para afirmar la pertenencia a la Unión, lo condujeron a llevar adelante la encuesta popular. Ni el Primer Ministro David Cameron, partidario de la permanencia, ni los principales referentes de las instituciones europeas y tal vez hasta los propios partidarios del

7 British Exit, o salida británica.

8 El referéndum realizado en el Reino Unido el 23 de junio de 2016 produjo el siguiente resultado: Votos a favor del Brexit: 17.410.742: Votos a favor de permanecer: 16.577.342; Total de votos: 33.577.342. Participación: 72\%. 
Brexit esperaban semejante el resultado.

Es probable que la suma de antiguas y reiteradas confrontaciones entre Bruselas y el Reino Unido, fueran generando suficiente predisposición entre los ciudadanos británicos más adultos, quienes votaron mayoritariamente por la salida, sin haber meditado en profundidad sobre los posibles efectos.

Fue también factor determinante la postura del ex alcalde de Londres Boris Johnson, que visualizó al mensaje Brexit como una oportunidad para ser Primer Ministro ${ }^{9}$, de igual modo la de Nigel Farage, reconocido euroescéptico quien se puso frontalmente a favor de la salida como parte de su política personal y partidaria ${ }^{10}$.

La renuncia de Cameron antes de iniciar el procedimiento para la salida, previsto en el artículo 50 del Tratado de Lisboa ${ }^{11}$, movilizó el proceso de su sustitución resultando la Ministra del Interior Theresa May electa en el cargo de Primer Ministro, a la misma corresponderá liderar las arduas decisiones que deberán ser adoptadas. .

Su postura firme respecto al problema de la inmigración y la adoptada frente al referendo la colocan en una posición inmejorable para ser la protagonista de las negociaciones nada fáciles que se avecinan y para intentar el reposicionamiento internacional del Reino Unido fuera de la Unión Europea.

\section{LA POSICIÓN DEL REINO UNIDO}

Si en su participación en la Unión Europea se analiza la posición Reino Unido, sobre todo los acuerdos obtenidos poco antes, es mucho más incomprensible el resultado del referéndum, si bien numerosas diferencias podrían haber alertar de algún modo sobre lo que sobrevendría.

El Reino Unido se ha presentado siempre como el miembro díscolo en el proceso de integración europeo, a raíz de posiciones encontradas con el objetivo de la unión como la no participación en el Acuerdo Schengen ${ }^{12}$, la Zona Euro, Política Exterior y de defensa común

9 Boris Johnson, al verificar el impacto ocasionado como consecuencia del Brexit, debió declarar rápidamente que no era candidato a Primer Ministro, renunciando a asumir parte de las consecuencias de su propia decisión. Se justificó diciendo "Debo decirles, mis amigos, que tras haber consultado con colegas y en vista de las circunstancias en el parlamento, he concluido que yo no puedo ser esa persona".

10 El principal promotor de la salida del Reino Unido, líder del UKIP o Partido de la Independencia del Reino Unido, renunció a la actividad partidaria manifestando que luego de recuperar el país pretendía recuperar su vida. Apuntó el resultado del referendo como una victoria que culmina todo lo anhelado por el mismo como político.

11 El Tratado de Lisboa entró en vigor el 1 de diciembre de 2009.

12 Por el Acuerdo de Scheng en varios países de Europa suprimieron los controles en las fronteras interiores (entre esos países) y trasladaron esos controles a las fronteras exteriores (con países terceros). El acuerdo, firmado en la ciudad luxemburguesa de Schengen en 1985 y en vigor desde 1995, establece un espacio común -denominado espacio de Schengenpor el que puede circular libremente toda persona que haya entrado regularmente por una 
y los reclamos permanentes sobre sus aportes o los beneficios recibidos. Sorprende sobre todo porque el Reino Unido antes del referéndum y como una garantía de que no se produciría la salida, había obtenido una suerte de "estatus especial" por el cual podía permanecer fuera del objetivo de una unión más estrecha o de toda posibilidad de formar parte de una federación europea.

Las demandas formuladas por el Reino Unido a Bruselas de limitar las ayudas sociales a los inmigrantes europeos, permanecer al margen de los próximos pasos hacia una mayor integración europea, mejorar la competitividad del mercado único y la protección de Londres respecto a las decisiones de la Eurozona, fueron aceptadas ${ }^{13}$.

Previo a la convocatoria del referéndum David Cameron efectuó una rueda de prensa en la cual aseguró que en virtud del acuerdo

frontera exterior o resida en uno de los países que aplican el Convenio. En total, los países que forman parte del espacio de Schengen son 26: Alemania, Austria, Bélgica, Dinamarca, Eslovaquia, Eslovenia, España, Estonia, Finlandia, Francia, Grecia, Hungría, Islandia, Italia, Letonia, Liechtenstein, Lituania, Luxemburgo, Malta, Noruega, Países Bajos, Polonia, Portugal, República Checa, Suecia y Suiza. Este último país votó el 9 de febrero de 2014 en referéndum, restringir el acceso de los ciudadanos de Europa a su territorio, lo que puede llevarle a abandonar el espacio de Schengen. Existen países que pertenecen al acuerdo de Schengen pero que tienen excepciones en la aplicación de algunos puntos del acuerdo y no pertenecen al espacio de Schengen. Por ejemplo, Reino Unido e Irlanda no forman parte del espacio de Schengen pero participan en la cooperación policial y judicial y en la lucha contra estupefacientes. Otros como Bulgaria, Rumanía y Chipre todavía no pertenecen al espacio de Schengen porque no cumplen con los requisitos de seguridad establecidos, aunque sí aplican otras partes del acuerdo. Islandia, Liechtenstein, Noruega y Suiza, que no son miembros de la UE, llegaron a un acuerdo para formar parte de la zona Schengen, aunque Suiza ha votado en referéndum abandonarlo. Croacia, último país en incorporarse a la UE, quiere integrarse en el acuerdo en 2015.

13 "Los jefes de Estado y de Gobierno de la UE llegaron a ese acuerdo tras dos intensas jornadas de negociaciones y después de que los líderes evaluaran en una cena a 28 el último proyecto de acuerdo. Ese incluye un compromiso entre el Reino Unido y los países del este de Europa en cuanto al freno de emergencia para restringir las prestaciones a trabajadores comunitarios y la indexación de las ayudas por hijo. En cuanto al primer punto, Reino Unido podrá restringir durante los cuatro primeros años de su contrato las prestaciones sociales a los trabajadores comunitarios y podrá recurrir a este mecanismo durante un periodo de siete años, sin prórrogas, indicaron fuentes diplomáticas. En lo que respecta a la indexación de los beneficios que los trabajadores comunitarios perciben por hijo, el acuerdo final recoge un periodo de transición de cuatro años, hasta 2020, para aquellos empleados que ya reciben una ayuda. El texto también establece principios para garantizar el respeto mutuo entre los Estados miembros que quieren avanzar en la profundización de la Unión Económica y Monetaria y los que no, como el Reino Unido, para lo que habrá un "mecanismo" mediante el que "un" país que no participa en el euro puede indicar su oposición razonada a una medida legislativa y elevarlo al Consejo. No obstante, las fuentes dejaron claro que el documento precisa que esta medida en ningún caso puede servir para vetar las decisiones de la eurozona y que el proceso tiene que producirse de manera urgente para no influir en los mercados. Las mismas fuentes diplomáticas también señalaron que para dirimir las diferencias en las interpretaciones sobre una "Unión cada vez más estrecha", el texto recoge que esta referencia no se aplica al Reino Unido, que no quiere una mayor integración política. También indicaron que en el texto final se menciona la posibilidad de introducir cambios en los tratados en el futuro para recoger algunos elementos del nuevo equilibrio con el Reino Unido." Reino Unido y la UE logran un acuerdo final dirigido a impedir el "Brexit". EFEBruselas, 19 feb 2016. Disponible en: <http://www.efe.com/efe/espana/portada/ reino-unido-y-la-ue-logran-un-acuerdo-final-dirigido-a-impedir-el-brexit/10010-284432> 
alcanzado "nunca nos uniremos al euro, ni formaremos parte de los rescates, ni de la Europa sin controles de pasaportes, ni del ejército europeo, ni de un super-estado europeo"14.

El presidente de la Comisión Europea, Jean-Claude Juncker se refirió a este acuerdo diciendo "Es justo con el Reino Unido, justo con los otros 27 Estados"15. "Es un buen compromiso, que espero que sirva para mantener a Gran Bretaña en la UE”, escribió el primer ministro checo, Bohuslav Sobotka. El presidente del Consejo Europeo, Donald Tusk manifestó "apoyo unánime para un nuevo encaje para el Reino Unido en la Unión Europea (UE)"16.

Frente a tal escenario, la posibilidad del Brexit era impensable, las condiciones reclamadas y obtenidas por Cameron previo al referéndum, aparentemente le garantizaban de que resultaba imposible que el Reino Unido abandonara la Unión Europea, sin embargo para sorpresa del propio Primer Ministro y de Bruselas, el referéndum votó por la salida de la Unión.

Las consecuencias son diversas y constituye una situación inédita para la Unión Europea, el Reino Unido, luego de Alemania y Francia es la tercera economía del bloque y como contribuyente el segundo en el presupuesto europeo, de allí que en el análisis de la salida y hasta que se concrete la Unión tendrá que prever una reingeniería presupuestaria.

En el proceso de salida las negociaciones serán arduas y complejas, la Unión Europea no puede perder de vista que semejante ejemplo, en la hipótesis que represente un claro y visible beneficio para el Reino Unido será una invitación a que otros Estados miembros, pretendan también un referéndum de continuidad.

La cuestión tiene múltiples aristas, desde analizar el interés económico de las partes, hasta el precedente que en uno u otro sentido pueden significar las negociaciones, por de pronto hay una especia de estado de conmoción y preocupación lógica sobre el futuro, abriendo las puertas a la posibilidad de una Unión Europea de múltiples velocidades.

Entre las negociaciones inevitables está la de si el Reino Unido podrá seguir participando o no del mercado único europeo, o en otros términos si podría o no mantenerse de alguna manera integrado a través del marco legal que representa el Espacio Económico Europeo "EEE"17, del cual participan actualmente Noruega, Liechtenstein e

14 La UE activaría el estatus especial para el Reino Unido si no hay Brexit. Diponible en: $<$ http://www.publico.es/internacional/ue-activaria-estatus-especial-reino.html>

15 Reino Unido y la UE logran un acuerdo final dirigido a impedir el "Brexit". EFE. Bruselas19 feb 2016. Disponible en: <http://www.efe.com/efe/espana/portada/ reino-unido-y-la-ue-logran-un-acuerdo-final-dirigido-a-impedir-el-brexit/10010-2844328>

16 Reino Unido y la UE logran un acuerdo final dirigido a impedir el 'Brexit'. Por EFE. Portada de RTVE. Disponible en: <http://www.rtve.es/noticias/20160219/reino-unido-uelogran-acuerdo-final-dirigido-impedir-brexit/1304980.shtml>

17 El Espacio Económico Europeo (EEE) se instauró el 1 de enero de 1994 con motivo de un acuerdo entre países miembros de la Unión Europea (UE) y de la Asociación Europea de Libre 
Islandia, manteniendo de este modo para los ciudadanos británicos y a su vez a los europeos la vigencia de las cuatro libertades que representa el mercado único.

En tal hipótesis, que podría resultar con diversos matices probable, la negociación podría mantener la legislación común relacionada al mercado único, o tendría la obligación de aceptarla, con lo cual estaría participando de uno de los principales mecanismos de integración del proceso europeo, sin incidir en su alcance pero todas las obligaciones de un Estado miembro.

Este escenario deberá soportar las críticas que supondría dicha situación al interior de la Unión y a las contradicciones que significaría, teniendo en cuenta que esencialmente el Brexit fue producto de un reclamo del rescate o mayor soberanía estatal del Reino Unido y el rechazo a las decisiones migratorias, que no podrían convivir con la libre circulación.

Otro escenario de negociación posible sería el que posee la Unión Europea con Suiza, cuya relación esta reglada por una multiplicad de acuerdos, aproximadamente cien, que regulan diversos intereses. El Reino Unido deberá definir en primer lugar cual es la vinculación que pretende mantener o busca establecer en las negociaciones, que podemos anticipar no es tarea fácil.

Entre los intereses que más pueden afectar al Reino Unido, está el rol que siempre ha desempeñado en el ámbito financiero, en el modelo de la relación de la Unión Europea con Suiza están excluidos los servicios financieros, servicio que es interés central británico. De lo que no cabe duda es que se requerirá mucha imaginación, poder de negociación y una apreciación objetiva de los intereses en juego para una salida airosa de esta difícil coyuntura para la integración europea.

La posibilidad de que el Reino Unido se mantenga absolutamente desvinculado de todo los acuerdos o de toda vinculación con la Unión Europea no parece ser viable, más allá de que se acepte o no, existen intereses que no pueden ser dejado de lado, como la relación estratégica que significa los vínculos existentes desde el punto de vista comercial, político y sobre todo social. No se debe olvidar el estrecho margen en la votación del referéndum y la presión que podría significar la pérdida de espacios y expectativas de los ciudadanos británicos.

Tal hipótesis, la de total desvinculación, supone que todas las negociaciones del Reino Unido se realizarán fuera de la protección

Comercio (EFTA), excepto Suiza. Su creación permitió a los países de la EFTA participar en el mercado interior de la Unión Europea sin tener que adherirse a la UE. Los miembros de la AELC siguientes: Islandia, Liechtenstein y Noruega. Suiza, como miembro de la AELC, rechazó por un 49,7\% entrar a formar parte del Espacio Económico Europeo en el referéndum nacional celebrado el 6 de diciembre de 1992 por lo que no ratificó el acuerdo. Actualmente las relaciones de ese país con la UE están regidas por un conjunto de tratados bilaterales como miembro de Schengen desde 2008. 
del marco regional y sujeto a las reglas de la Organización Mundial de Comercio, lo que llevará a una revisión muy profunda de sus relaciones comerciales internacionales y el riesgo previsible de que se presente en condiciones menos ventajosa frente a los Estados que poseen acuerdos comerciales con la Unión Europea.

Otra cuestión no menor, es la oportunidad o no de reformar los Tratados a fin de prever situaciones como las que se presentan con el Reino Unido, para cuyo efecto habrá que analizar muy especialmente la coyuntura política y necesidades que se definan como prioridades futuras, como el reconocimiento expreso de una Europa a velocidades distintas o avanzar hacia un sistema federativo.

Los comentarios de los medios, ante la falta de referencias de otro tipo son múltiples y algunos, como el The Guardian exponen sobre las posibles consecuencias "La decisión histórica del Reino Unido de terminar la relación de amor y odio con la Unión Europea comenzada hace 43 años representa un punto de inflexión en la historia británica tan importante como las dos guerras mundiales del siglo XX. Suponiendo que no hay vuelta atrás, ni un repentino arrepentimiento colectivo, las consecuencias políticas, constitucionales, diplomáticas y económicas para el Reino Unido se extenderán al menos durante una década.

La posición del Reino Unido en el mundo acaba de cambiar, como también han cambiado los centros de poder de la política británica. Downing Street, las grandes corporaciones, los expertos económicos o el establishment de la política exterior, los tradicionales centros de poder, acaban de ser golpeados por el equivalente a una bomba de racimo popular"18.

Consecuencias indirectas del Brexit es de que podría haber generado las condiciones para una nuevo consulta sobrela independencia de Escocia ${ }^{19}$, que en un $62 \%$ por ciento se pronunciaron en favor de permanecer en la Unión Europea, es previsible que el resultado pudiera reactivar la posibilidad de un nuevo referéndum de independencia del Reino Unido de modo a permanecer en la Unión Europea ${ }^{20}$. Lo mismo

18 ¿Qué ocurrirá ahora en Reino Unido y Europa tras el sí al Brexit?. Por Theguardian. Eldiario.es. Disponible en: <http://www.eldiario.es/theguardian/Reino-Unido-UnionEuropea-ahora_0_530197283.html>

19 "Nicola Sturgeon, primera ministra escocesa y líder del independentista Partido Nacional Escocés (SNP), declaró que un segundo referéndum de independencia era "altamente probable". El líder del partido republicano irlandés Sinn Fein, Martin McGuinness, reclamaba esa misma mañana que se dejara "a la gente del Norte tener voz sobre su propio futuro". Escocia podría de esta forma estar avanzando hacia su segundo referéndum de independencia, después del celebrado en el 2014. A pesar de que los escoceses rechazaron entonces separarse del Reino Unido, el apoyo por la independencia ha crecido desde entonces y el SNP se ha mantenido como fuerza política hegemónica en Escocia en las dos últimas elecciones.”. TRAS LA VICTORIA DEL 'BREXIT' Irlanda del Norte y Escocia exigen sendos referéndums para salir de Gran Bretaña. El Periódico Internacional. Disponible en: <http://www.elperiodico.com/es/ noticias/internacional/escocia-irlanda-del-norte-votan-favor-seguir-union-europea-5226085 $20>$. En la primera consulta, en 2014, la opción de permanecer en Reino Unido se impuso sólo 
cabe decir de Irlanda del Norte.

El procedimiento para que se efectivice la salida está sujeto a lo previsto en el artículo 50 del Tratado de Lisboa. Es posible anticipar que se buscará generar una nueva y particular relación entre el bloque y el Reino Unido que ampare de algún modo a quienes ya se encuentran con derechos derivados de los acuerdos pre existentes y establezca el escenario económico para las exportaciones e importaciones futuras tanto con el bloque como con relación a terceros estados, con los cuales se celebraran acuerdos comerciales ${ }^{21}$.

La activación del proceso de salida corresponde al Estado miembro interesado y por tanto deberá ser el Reino Unido que lo active por medio de la notificación. Conforme a su constitución corresponde al Primer Ministro y no al Parlamento hacerlo, por cuanto constituye una prerrogativa real, aun cuando puede proponer una moción de que el Primer Ministro no active el artículo 50 del Tratado de Lisboa.

Una vez iniciado el procedimiento por la comunicación que haga la nueva Primer Ministro británica Theresa May, la negociación o acuerdo al cual se arribe para su aprobación precisa de una mayoría calificada de votos tanto del Reino Unido como de los demás veinte y siete miembros, lo que representa el voto favorable de veinte países y por lo menos el $65 \%$ de la población total.

De no producirse el acuerdo y no se llegare a la mayoría para su aprobación o un acuerdo de prórroga o extensión, todas las relaciones comerciales del Reino Unido con la Unión Europea serán regidos por las reglas y aranceles en la Organización Mundial de Comercio y quedará fuera de todos los acuerdos celebrados.

\section{EL ARTÍCULO 50 DEL TRATADO DE LISBOA}

La Unión Europea ha previsto en el artículo 50 del Tratado de la Unión Europea un mecanismo para la retirada voluntaria y unilateral de un Estado Miembro de la Unión Europea (UE), disposición a la cual se refiere como "Cláusula de retirada".

El procedimiento consiste, en la notificación que realiza el Estado que adopta la decisión de retirarse al Consejo Europeo, a lo cual sigue una negociación a fin de que finalmente el Consejo por mayoría calificada y previa aprobación del Parlamento Europeo, proponga las directrices para la celebración del acuerdo de retirada.

Los Tratados dejan de aplicarse para quien realiza la solicitud a

con un 55\% de los votos contra el 44\% que apoyó la separación.

21 La mitad de las exportaciones de Reino Unido se dirigen a día de hoy hacia la Unión, mientras que el país británico recibe hasta un $16 \%$ de las exportaciones europeas. Pero la UE afecta también al modo en que Reino Unido comercia con el resto del mundo: gran parte de los intercambios comerciales del país británico se desarrollan en el marco de los acuerdos que la Unión tiene con 50 países, acuerdos que tendrían que ser renegociados. 
partir de la entrada en vigor del acuerdo o a más tardar, aun no mediando acuerdo, a partir de los dos años de la notificación de retirada, plazo que puede ser prorrogado por el Consejo. El Estado saliente podrá volver a solicitar su ingreso a la Unión, pero deberá seguir todo el procedimiento previsto para la adhesión.

Es posible suponer que al tiempo de la redacción del artículo 50 del Tratado de Lisboa, no se habría pensado que los referentes más importantes de la Unión Europea utilizarían el mecanismo de salida, teniendo en cuenta la pérdida de representatividad, del espacio económico y tener que actuar aislado en un mundo definitivamente globalizado.

Tal vez si se hubiera visualizado más profundamente esa situación, la redacción del texto hubiera sido diferente, contemplando mecanismos que permitieran un procedimiento más lento y la posibilidad de revisar la decisión o incluso una reincorporación más simple, pero no es así.

En su texto el inciso primero es terminante, acompaña en realidad lo que constituye un derecho fundamental de todo Estado de permanecer o no ligado a un tratado cuyos objetivos o marco legal ya no lo satisfacen o son cuestionados. Dice "1. Todo Estado miembro podrá decidir, de conformidad con sus normas constitucionales, retirarse de la Unión.”.

La redacción obedece al principio de que la adhesión de los Estados a la Unión Europea es un acto voluntario y soberano, por lo cual cada uno de los miembros puede decidir su retiro. Igualmente a que ante semejante decisión, lo más aconsejable resulte acelerar la separación a fin de no afectar las políticas comunes.

La disposición es simple y al mismo tiempo impactante porque implica la separación o escisión de un miembro del espacio integrado y de los acuerdos que permitieron la construcción de una comunidad jurídica, lógicamente con las consecuencias políticas que tal decisión representa también para los demás miembros.

Es una decisión autónoma que puede adoptar cada Estado miembro conforme sus propios preceptos constitucionales, de allí que habrá mayores o menores dificultades para la decisión según la variedad de preceptos constitucionales. En el caso del Reino Unido, basta el referéndum y este fue realizado votando por la salida de la Unión Europea, decisión que parece irreversible y que da inicio a la separación a partir de la en la comunicación que se efectúe de la decisión adoptada en el mismo.

Si nos remitimos para el análisis a la Convención de Viena sobre el Derecho de los Tratados ${ }^{22}$, la terminación de un tratado o el retiro se produce en virtud de sus disposiciones o por el consentimiento de las partes. El Art 54 en el inciso a) de la Convención, refiere que se produce “a) conforme a las disposiciones del tratado, o b) en cualquier momento,

22 Convención de Viena sobre el derecho de los tratados. U.N. Doc A/CONF.39/27 (1969), 1155 U.N.T.S. 331, entered into force January 27, 1980. Viena, 23 de mayo de 1969. 
por consentimiento de todas las partes después de consultar a los demás Estados contratantes".

En el caso de la Unión Europea, conforme al artículo 50, la decisión queda abierta a todos los Estados miembros, siguiendo sus normas constitucionales, que es precisamente lo que ha efectuado el Reino Unido a través del referéndum. La disposición protege el derecho a no seguir tomando parte en la estructura jurídico-política de la Unión Europea como una garantía para los Estados miembros, a fin de evitar quedar sujetos a situaciones no deseadas.

El Tratado de la Unión celebrado que sea un acuerdo internacional entre la Unión ${ }^{23}$ y el Estado que se constituye en miembro, por tanto prevé el mecanismo para el Estado que resolviere retirarse, intención que debe ser notificada al Consejo Europeo ${ }^{24}$. A partir de este momento la Unión debe negociar y celebrar un acuerdo para determinar la forma de retirada atendiendo a las relaciones futuras con el Estado saliente ${ }^{25}$.

La disposición remite al artículo 218.3 del Tratado de Funcionamiento de la Unión Europea (antiguo artículo 300 TCE) sobre la forma de negociación, interpretando esta remisión, se trata de una negociación destinada a reglar la relación futura del Estado que se retira y la Unión Europea.

La remisión legislativa se refiere a negociaciones con terceros Estados, de lo cual se deduce que más allá de la continuidad de las obligaciones recíprocas que se extienden por dos años, salvo acuerdo de prórroga, las autoridades de la Unión deben asumir que se trata de

23 Artículo 216 del Tratado de Funcionamiento de la Unión Europea "1. La Unión podrá celebrar un acuerdo con uno o varios terceros países u organizaciones internacionales cuando así lo prevean los Tratados o cuando la celebración de un acuerdo bien sea necesaria para alcanzar, en el contexto de las políticas de la Unión, alguno de los objetivos establecidos en los Tratados, bien esté prevista en un acto jurídicamente vinculante de la Unión, o bien pueda afectar a normas comunes o alterar el alcance de las mismas. 2. Los acuerdos celebrados por la Unión vincularán a las instituciones de la Unión y a los Estados miembros.”

24 El Consejo Europeo es una de las siete altas Instituciones de la Unión Europea, integrada por los veintiocho jefes de Estado o de Gobierno de los países miembros, el Presidente de la Comisión Europea, y el presidente del Consejo Europeo, que es quien preside las reuniones. En sus reuniones y trabajos también participa el Alto Representante de la Unión para Asuntos Exteriores y Política de Seguridad, aun no siendo miembro de pleno derecho. Su composición y la lógica de su funcionamiento lo convierten en un órgano de naturaleza predominantemente intergubernamental.1 Sus funciones son de orientación política y de jefatura colectiva simbólica, fijando las grandes directrices y objetivos de la Unión en los ámbitos más relevantes; la potestad legislativa le está expresamente vedada por los Tratados. Sus reuniones, de carácter ordinariamente trimestral, se designan comúnmente como "cumbres europeas" o "reuniones en la cumbre" debido a la importancia de sus asistentes, pero desde la entrada en vigor del Tratado de Lisboa, el $1^{\circ}$ de diciembre de 2009, el Consejo Europeo es una institución constitucional comunitaria de pleno Derecho y como tal debe actuar, al servicio del acuerdo e integración de los intereses nacionales con los europeos.

25 Artículo 218 del Tratado de Funcionamiento de la Unión Europea (antiguo artículo 300 TCE). 1. Sin perjuicio de las disposiciones particulares del artículo 207, para la negociación y celebración de acuerdos entre la Unión y terceros países u organizaciones internacionales se aplicará el procedimiento siguiente. 2. El Consejo autorizará la apertura de negociaciones, aprobará las directrices de negociación, autorizará la firma y celebrará los acuerdos. 
una negociación para un acuerdo futuro con un tercero ${ }^{26}$. La Unión negocia a través del Consejo con el Estado que comunicó su decisión de retirarse, lo hace conforme al citado artículo 218.3 del TFUE.

De acuerdo al artículo 50 del Tratado de Lisboa "La Comisión o el Alto Representante de la Unión para Asuntos Exteriores y Política de Seguridad, presentará recomendaciones al Consejo, que adoptará una decisión por la que se autorice la apertura de negociaciones y se designe al negociador o al jefe del equipo de negociación de la Unión". Siempre en virtud del artículo 50 del TU "El Consejo celebrará el acuerdo en nombre de la Unión por mayoría cualificada, previa aprobación del Parlamento Europeo".

De acuerdo al artículo 218 del Tratado de Funcionamiento de la Unión Europea inciso 2. "El Consejo autorizará la apertura de negociaciones, aprobará las directrices de negociación, autorizará la firma y celebrará los acuerdos", teniendo en cuenta que la negociación con el Reino Unido implicará asuntos exteriores y política de seguridad, tanto la Comisión, o el Alto Representante de la Unión para Asuntos y Exteriores y Política de Seguridad, presentarán recomendaciones al Consejo.

Le corresponde al Consejo designar, teniendo en vista la materia del acuerdo previsto, al negociador o al jefe del equipo de negociación de la Unión. También dictar directrices al negociador y designar un comité especial, al que deberá consultarse durante las negociaciones. (inciso 4).

De acuerdo al numeral 5 del artículo 218.3 del TFUE "El Consejo adoptará, a propuesta del negociador, una decisión por la que se autorice la firma del acuerdo y, en su caso, su aplicación provisional antes de la entrada en vigor". Se debe tener presente que El Consejo adoptará, a propuesta del negociador, una decisión de celebración del acuerdo" (numeral 6).

Siempre en base al numeral 6 el Consejo adoptará la decisión de celebración del acuerdo, previa aprobación del Parlamento Europeo (6.a) en los siguientes casos. $i$. acuerdos de asociación; ii. acuerdo de adhesión de la Unión al Convenio Europeo para la Protección de los Derechos Humanos y de las Libertades Fundamentales; iii. acuerdos que creen un marco institucional específico al organizar procedimientos de cooperación; $i v$. acuerdos que tengan repercusiones presupuestarias importantes para la Unión; v. acuerdos que se refieran a ámbitos a los que se aplique el procedimiento legislativo ordinario o, si se requiere la

26 Artículo 218 del Tratado de Funcionamiento de la Unión Europea (antiguo artículo 300 TCE) 1. Sin perjuicio de las disposiciones particulares del artículo 207, para la negociación y celebración de acuerdos entre la Unión y terceros países u organizaciones internacionales se aplicará el procedimiento siguiente. Artículo 207. Tratado de Funcionamiento de la Unión Europea (antiguo artículo 133 TCE) 3. En caso de que deban negociarse y celebrarse acuerdos con uno o más terceros países u organizaciones internacionales, se aplicará el artículo 218 , sin perjuicio de las disposiciones específicas del presente artículo. 
aprobación del Parlamento Europeo, el procedimiento legislativo especial.

En caso de urgencia, el Parlamento Europeo y el Consejo podrán convenir en un plazo para la aprobación previa consulta al Parlamento Europeo en los demás casos. El Parlamento Europeo emitirá su dictamen en un plazo que el Consejo podrá fijar según la urgencia. De no haberse emitido un dictamen al término de dicho plazo, el Consejo podrá pronunciarse.

Lo dispuesto precedentemente tiene por excepción los acuerdos que se refieran exclusivamente a la política exterior y de seguridad común, que hemos visto tiene un trámite especial contenido también en el artículo 50 del Tratado de Lisboa.

El consejo, de acuerdo al numeral 7 "podrá autorizar al negociador a aprobar, en nombre de la Unión, las modificaciones del acuerdo para cuya adopción éste prevea un procedimiento simplificado o la intervención de un órgano creado por el acuerdo. El Consejo podrá supeditar dicha autorización a condiciones específicas".

En cuanto al modo de pronunciamiento de acuerdo al numeral 8 "El Consejo se pronunciará por mayoría cualificada durante todo el procedimiento. Sin embargo, el Consejo se pronunciará por unanimidad cuando el acuerdo se refiera a un ámbito en el que se requiera la unanimidad para la adopción de un acto de la Unión y cuando se trate de acuerdos de asociación y de los acuerdos previstos en el artículo 212 con los Estados candidatos a la adhesión. El Consejo se pronunciará también por unanimidad sobre el acuerdo de adhesión de la Unión al Convenio Europeo para la Protección de los Derechos Humanos y de las Libertades Fundamentales; la decisión de celebración de dicho acuerdo entrará en vigor después de haber sido aprobada por los Estados miembros, de conformidad con sus respectivas normas constitucionales.

Conforme al numeral 9. "El Consejo adoptará, a propuesta de la Comisión o del Alto Representante de la Unión para Asuntos Exteriores y Política de Seguridad, una decisión por la que se suspenda la aplicación de un acuerdo y se establezcan las posiciones que deban adoptarse en nombre de la Unión en un organismo creado por un acuerdo, cuando dicho organismo deba adoptar actos que surtan efectos jurídicos, con excepción de los actos que completen o modifiquen el marco institucional del acuerdo".

Por último, numeral 10. "Se informará cumplida e inmediatamente al Parlamento Europeo en todas las fases del procedimiento". De acuerdo al 11 "Un Estado miembro, el Parlamento Europeo, el Consejo o la Comisión podrán solicitar el dictamen del Tribunal de Justicia sobre la compatibilidad con los Tratados de cualquier acuerdo previsto. En caso de dictamen negativo del Tribunal de Justicia, el acuerdo previsto no podrá entrar en vigor, salvo modificación de éste o revisión de los Tratados". 
De acuerdo al numeral 2 del artículo 50 del Tratado de Lisboa, el período de negociación a partir de la notificación es de dos años, pasado los cuales, salvo que el Consejo Europeo amplíe el plazo, los "Los Tratados dejarán de aplicarse al Estado de que se trate a partir de la fecha de entrada en vigor del acuerdo de retirada o, en su defecto, a los 2 años de la notificación de retirada al Consejo Europeo. El miembro del Consejo Europeo y del Consejo que represente al Estado miembro que se retire no participará ni en las deliberaciones ni en las decisiones del Consejo Europeo o del Consejo que le afecten".

La mayoría cualificada, se definirá como un mínimo del 72\% de los miembros del Consejo que represente a Estados miembros participantes que reúnan como mínimo el 65\% de la población de dichos estados. Si el estado miembro que se ha retirado de la Unión solicita de nuevo la adhesión, su solicitud se someterá al procedimiento establecido en el artículo 49 del Tratado de la Unión Europea.

\section{PETICIÓN DE READHESIÓN}

La hipótesis de una nueva adhesión del Reino Unido una vez culminado el procedimiento de retiro, no tendrá privilegios especiales, debe seguir el procedimiento previsto en el artículo 49 del TUE, que establece la base para este efecto y cumplir los criterios de elegibilidad, entre ellos el del artículo 2 del TUE como el respeto de la dignidad humana, la libertad, la democracia, la igualdad y el Estado de Derecho; el respeto de los derechos humanos, incluidos los derechos de las personas pertenecientes a minorías; y el respeto de una sociedad pluralista, así como la no discriminación, la tolerancia, la justicia, la solidaridad y la igualdad entre mujeres y hombres.

También deberá cumplir con los denominados criterios de Copenhague, como fue denominado en razón de la reunión del Consejo Europeo que se celebrado en esa ciudad en 1993 como la existencia de instituciones estables que garanticen la democracia, el Estado de Derecho, el respeto de los derechos humanos y el respeto y la protección de las minorías; la existencia de una economía de mercado en funcionamiento y la capacidad de hacer frente a la competencia y las fuerzas del mercado dentro de la UE; la capacidad para asumir y cumplir de manera eficaz las obligaciones que se derivan de la adhesión, incluidos los objetivos de la unión política, económica y monetaria.

Es obvio que estos criterios reúne el Reino Unido, quizás se le pudiera objetar en tal hipótesis el relativo a la capacidad de cumplir eficazmente los objetivos principales de unión política, económica y monetaria, pero no debería ser un obstáculo. El Consejo Europeo realizado en Madrid en 1995 agregó que el candidato debe aplicar y garantizar la aplicación del Derecho de la Unión Europea por su trasposición a la legislación nacional 
y es la Unión Europea por supuesto la que se reserva decidir cuándo los criterios están cumplidos para la adhesión.

La petición de nueva adhesión será presentada al Consejo de la Unión Europea y este informará al Parlamento, a la Comisión Europea y a los Parlamentos Nacionales. El Consejo en tal caso concede al Estado el estatus de país candidato una vez que la Comisión dictamine favorable. Sólo luego de la decisión del Consejo que debe ser unánime se inician las negociaciones realizadas como conferencias intergubernamentales entre los Estados que conforman la Unión Europea y el país candidato.

Las negociaciones con conducen al proyecto de Tratado de Adhesión que debe ser aprobada por unanimidad del Consejo de la Unión Europea y tener la aprobación del Parlamento Europeo, luego de lo cual cada estado miembro y el país adherente los suscriben y ratifican.

\section{LA ALTERNATIVA DE INTEGRAR EL EEE}

Entre las alternativas posibles dentro del marco de negociación se encuentra la de que el Reino Unido se incorporare a la Asociación Europea de Libre Comercio o "AELC"27, cuyo origen fue propulsado por los británicos, esquema incorporado o integrado al Espacio Económico Europeo "EEE"28.

De acuerdo al artículo 1 del EEE su objetivo es el de promover un reforzamiento continuo y equilibrado de las relaciones comerciales y económicas entre las Partes Contratantes, en igualdad de condiciones de competencia y en observancia de unas normas comunes, con miras a crear un Espacio Económico Europeo homogéneo ${ }^{29}$.

27 La European Free Trade Association "EFTA" o EFTA fue firmado el 4 de enero de 1960 bajo la idea de servir de contrapeso a la CEE. Sus miembros originarios Reino Unido, Suecia, Noruega, Dinamarca, Suiza, Austria y Portugal y posteriormente Islandia y Finlandia. El Reino Unido ingresó en 1972 al Mercado Común y posteriormente otros de sus miembros. En 1991 se acordó una Zona de Libre Comercio con la Comunidad Económica Europea que entró en vigor en 1993. En 1992 el EFTA se incorpora al Espacio Económico Europeo EEE y sus miembros son Noruega, Islandia y Liechteintein.

28 Órganos del Espacio Económico Europeo. Consejo Europeo: Es el órgano encargado de impulsar la aplicación y desarrollo del Acuerdo y su evaluación. Dicho Consejo, que se reúne cada seis meses, está compuesto por los ministros competentes en cada materia de cada una de las dos comunidades: la UE y la EFTA. Las decisiones están adoptadas de común acuerdo. Comité Conjunto: Órgano dependiente del Consejo del EEE que se ocupa de las cuestiones prácticas y de la gestión ordinaria. Autoridad de Vigilancia:

Órgano que supervisa el cumplimiento de la normativa relativa al Espacio Económico Europeo.

El EEE cuenta además con dos órganos consultivos: un Comité Parlamentario, integrado por miembros de los Parlamentos de los países miembros, y un Comité Consultivo compuesto por miembros del Comité Económico y Social de la UE y por miembros del Comité Consultivo de la EFTA.” Véase: Biografía de Espacio Económico Europeo (EEE). Disponible en: $<$ http://www. lahistoriaconmapas.com/historia/historia2/biografia-de-espacio-economico-europeo-eee/> 29 "El EEE, sin embargo, difiere del mercado único comunitario en que no comprende todos los sectores políticos de la UE; la política agrícola común (PAC) y la política pesquera común son los principales sectores excluidos en principio del ámbito del Acuerdo. El EEE no cubre los 
El AELC ${ }^{30}$ preveía el establecimiento de un mercado interior que comprendiera la entonces Comunidad Europea "CE" y sus Estados miembros y los países del que forman parte del mismo.

Para el ingreso del Reino Unido será necesario que a más Islandia, Noruega, Liechtenstein y Suiza que tiene una relación especial con la Unión Europea acepten el ingreso. Noruega ya ha manifestado con anterioridad al referéndum que tal hipótesis no sería aceptable $e^{31}$.

En principio esta opción podría ser la que más se adecue para una relación entre la Unión Europea y el Reino Unido, analizar la posibilidad de integrar el Espacio Económico Europeo "EEE" al cual ingreso EFTA es una alternativa interesante, pero no exenta de obstáculos.

El principal obstáculo es que por este acuerdo pueden comerciar libremente en todo el espacio de la Unión Europea, pero están obligados a ser parte del acuerdo Schengen relacionado a la libre circulación de personas, uno de los puntos fundamentales de oposición esgrimidos para el voto favorable a la salida del Reino Unido, otro es el de los aportes que requiere

Noruega sería partidario de que el Reino Unido efectúe una negociación bilateral diferente, buscando preservar la relación que viene funcionando sin sobresaltos ${ }^{32}$, pero pese que es un socio comercial importante de noruega y del EFTA se manifiesta que éste sea el escenario de negociación. Sea como fuere el Reino Unido puede negociar un acuerdo similar al EFTA y tener presente todo el contenido del Espacio

impuestos indirectos ( IVA e impuestos especiales), ni tampoco tiene una política económica y comercial exterior común (arancel aduanero común, medidas antidumping, etc.).” Véase "El Espacio Económico Europeo (EEE) y la ampliación de la Unión Europea”. Disponible en: <http://www.europarl.europa.eu/enlargement/briefings/32a2_es.htm>

30 El Acuerdo se firmó el 2 de mayo de 1992 en Oporto entre los 12 Estados miembros de entonces de la CE y los 7 miembros de la AELC: Austria, Finlandia, Islandia, Liechtenstein, Noruega, Suecia y Suiza. Suiza, sin embargo, no pudo ratificar el Acuerdo debido al resultado negativo, por muy pequeño margen, del referéndum celebrado en diciembre de 1992. A causa de su unión económica de facto, Liechtenstein quedó inicialmente vinculado a Suiza. El Acuerdo entró en vigor a principios de 1994, por tanto, con 17 países. Desde entonces, tres de los miembros por parte de la AELC, Austria, Finlandia y Suecia, son ya miembros de pleno derecho de la Unión Europea. El principado de Liechtenstein consiguió adherirse al EEE el 1 de mayo de 1995, aunque con la condición de efectuar ciertas adaptaciones del acuerdo de unión aduanera de 1993 entre los dos Estados. El Acuerdo EEE, por lo tanto, se aplica ahora a la UE de los 15 y a los 3 Estados miembros de la AELC: Islandia, Liechtenstein y Noruega. Suiza, aunque no forma parte del EEE, sigue siendo miembro de la AELC.

31 Tras el Brexit, la EFTA es una opción para el Reino Unido, pero Noruega se opone a su entrada. El Economista.es/Bloomberg, BLOOMBERG 1/07/2016. Disponible en: <http://www. eleconomista.es/economia/noticias/7677327/07/16/Tras-el-Brexit-la-EFTA-es-una-opcionpara-el-Reino-Unido-pero-Noruega-se-opone.html>

32 Jonas Gahr Stoere, líder del partido laborista noruego, cree que lo mejor es que el Reino Unido fabrique su propia vía para tener acceso al mercado común de la UE. Noruega debe garantizar y asegurar la estabilidad de la EFTA. "Hay pocas posibilidades de que lleguemos a un acuerdo para cambiar algo que lleva funcionando más de 20 años", explicó el político noruego. Ver: Tras el Brexit, la EFTA es una opción para el Reino Unido, pero Noruega se opone a su entrada. ELECONOMISTA.ES / BLOOMBERG 1/07/2016. Disponible en: <http:// www.eleconomista.es/economia/noticias/7677327/07/16/Tras-el-Brexit-la-EFTA-es-unaopcion-para-el-Reino-Unido-pero-Noruega-se-opone.html> 
Económico Europeo.

\section{EL MODELO UNIÓN EUROPEA - SUIZA}

Entre los diversos escenarios que podrían ser tenidos en cuenta para el futuro de la relación de la Unión Europea con el Reino Unido, se podría tomar en consideración o referencia establecido con la Confederación Suiza ${ }^{33}$, pero con ciertas dificultades a raíz de la posición asumida con relación a la inmigración de ciudadanos europeos y las condiciones en las que esta podría producirse.

Según este modelo tanto los ciudadanos europeos como británicos, gozarían del derecho de entrada y residencia, acceso a una actividad económica, establecimiento como trabajador independiente y residencia una vez finalizada la actividad. El derecho de entrada y residencia afecta a todas las personas, incluidas aquellas que no ejerzan ninguna actividad económica en el país de acogida.

Implica que el Reino Unido deberá tratar a los ciudadanos europeos y a los británicos en el territorio de la Unión, bajo las mismas condiciones de vida, empleo y trabajo que a sus nacionales, básicamente el respeto a la libre circulación de personas, su movilidad y la apertura para ejercer actividades económicas y establecer residencia adquiriendo inmuebles.

En un acuerdo de esta naturaleza, el principal componente será la igualdad de trato, un sistema de coordinación en los sistemas de seguridad social y el reconocimiento de las cualificaciones profesionales.

Respecto a las prestaciones de servicios, quizás la mayor preocupación de las empresas que se han constituido para este efecto en el Reino Unido o las empresas británicas constituidas en el territorio de Estados de la Unión, la forma de evitar consecuencias económicas sería seguir el mismo modelo.

En tal sentido, deberían poder efectuar prestaciones limitadas y negociadas en cuanto a su duración y contemplar el modo de preservar los derechos y situación jurídica de las empresas ya instaladas.

33 Decisión 2002/309/CE, Euratom del Consejo y de la Comisión respecto al Acuerdo de cooperación científica y tecnológica de 4 de abril de 2002 sobre la celebración de siete Acuerdos con la Confederación Suiza [Diario Oficial L 114 de 30.4.2002]. Acuerdo entre la Comunidad Europea y sus Estados miembros, por una parte, y la Confederación Suiza, por otra, sobre la libre circulación de personas [DO L 114 de 30.4.2002]. Véase igualmente 2006/245/CE: Decisión del Consejo, de 27 de febrero de 2006 , relativa a la celebración, en nombre de la Comunidad Europea y de sus Estados miembros, de un Protocolo del Acuerdo entre la Comunidad Europea y sus Estados miembros, por una parte, y la Confederación Suiza, por otra, sobre la libre circulación de personas, relativo a la participación, como Partes Contratantes, de la República Checa, la República de Estonia, la República de Chipre, la República de Letonia, la República de Lituania, la República de Hungría, la República de Malta, la República de Polonia, la República de Eslovenia y la República Eslovaca, como consecuencia de su adhesión a la Unión Europea 


\section{CONCLUSIÓN}

1. La primera experiencia de la Unión Europea donde uno de sus miembros más destacados, el Reino Unido, decide salir de la Unión es un hecho absolutamente inesperado, sorprendió a la mayoría de los analistas del proceso de integración y a los propios referentes del mismo. Es una coyuntura inédita y el análisis de tal situación no es simple.

2. La situación exige de la Unión Europea un mensaje claro de que esta escisión no importará el resquebrajamiento del proceso de integración, ni su flexibilización, deberá adoptar medidas necesarias que fortalezcan el proceso para que no disminuya su fortaleza internacional.

3. Las negociaciones, que no serán fáciles, deberían ubicar al ReinoUnido en un estatus especial a través de un Tratado. Resulta aconsejable porsu particular condición, teniendo en cuenta intereses comunes, derechos adquiridos y la necesidad de mantener ciertas políticasregionales comunes, así lo exigen y es evidente que buscará el mayoracceso posible al mercado único.

4. Las negociaciones a la luz del artículo 50 del Tratado de Lisboa otorgan al Consejo Europeo amplias facultades. Esta negociación, debería iniciarse cuanto antes, a fin de terminar con las especulaciones y sondear las posibilidades de un acuerdo que permita una beneficiosa convivencia política y económica.

5. Es previsible que se haga uso del mecanismo de prórroga o extensión del plazo, sea parcial o total, que posibilita el artículo 50 numeral 3 del Tratado de Lisboa, a fin de culminar de forma adecuada y satisfactoria la negociación de salida o de nueva relación entre el Reino Unido y la Unión Europea.

6. Durante las negociaciones, hasta arribar a un acuerdo o durante el período de dos años, el Reino Unido sigue siendo un miembro con todas las obligaciones y derechos que implica la condición de miembro y de ciudadanos de la Unión. Luego de ese período sobrevendrán, cuestiones vinculadas a los derechos adquiridos o consolidados de los ciudadanos de la Unión Europea y del Reino Unido, que deberán ser resueltos en la medida en que sean planteados en favor de su reconocimiento, para evitar que decisiones políticas incidan sobre situaciones preexistentes de naturaleza personal y comercial.

7. La negociación será una experiencia inédita, las partes deberán analizar y sopesar sus planteamientos. Obviamente el Reino Unido buscara mantener en gran medida el acceso al mercado interior, sin las responsabilidades que implica, en tanto que la Unión Europea condicionara el acceso a la contribución que considere necesaria, a fin de evitar un socio "privilegiado" que obtenga todos los beneficios sin contribuir al presupuesto.

8. Es previsible que las negociaciones respecto al acceso al 
mercado por el Reino Unido, se garantizará que no resulten en una discriminación respecto a los Estados miembros de la Unión Europea. Es probable, en el futuro, que las obligaciones acordadas o extendidas sobre el mercado único deberán ser respetadas por el Reino Unidosin intervenir en la elaboración, modificación de la legislación comunitaria.

9. Los escenarios previsibles para una relación futura entre el Reino Unido y la Unión Europea puede seguir diversos modelos, como el de incorporación al Espacio Económico Europeo vía Asociación Europea de Libre Comercio o "AELC", lo que implica para el Reino Unido formar parte del mercado único europeo sin restringir la posibilidad de negociar con terceros países, sin voto en las decisiones y la obligación de contribuir a la UE.

La otra alternativa es adoptar un acuerdo similar al de Suiza, implica libre circulación de personas y bienes, sin toma de decisiones y la escisión total por lo que los acuerdos serán regidos bajo las reglas de la Organización Mundial de Comercio.

Por su parte, independientemente a esta negociación, la Unión Europea debería profundizar la idea de una integración de varias velocidades, a fin de evitar que situaciones de disconformidad o el deseo de no avanzar con nuevas concesiones o cesión de poderes a las instituciones, trabe el proceso o produzca situaciones similares a las del Reino Unido.

\section{REFERENCIAS BIBLIOGRÁFICAS}

“¿Qué ocurrirá ahora en Reino Unido y Europa tras el sí al Brexit?” [en línea]. Eldiario.es. 24 jun 2016. Disponible en: <http://www.eldiario.es/ theguardian/Reino-Unido-Union-Europea-ahora_0_530197283.html>

"El Espacio Económico Europeo (EEE) y la ampliación de la Unión Europea" [en línea]. Europa Parliament. 17 nov 1998. Disponible en: $<$ http://www.europarl.europa.eu/enlargement/briefings/32a2_es.htm>

"Espacio Económico Europeo" [en línea]. La Historia.com. 1 nov 2007. Disponible en: <http://www.lahistoriaconmapas.com/historia/ historia2/biografia-de-espacio-economico-europeo-eee/>

"La UE activaría el estatus especial para el Reino Unido si no hay Brexit" [en línea]. Público. 22 jun 2013. Disponible en: < http://www.publico.es/ internacional/ue-activaria-estatus-especial-reino.html $>$

"Reino Unido y la UE logran un acuerdo final dirigido a impedir el "Brexit"' [en línea]. Agencia EFE. 19 feb 2016. Disponible en: <http:// www.efe.com/efe/espana/portada/reino-unido-y-la-ue-logran-unacuerdo-final-dirigido-a-impedir-el-brexit/10010-2844328>

"Reino Unido y la UE logran un acuerdo final dirigido a impedir el 'Brexit"' [en línea]. Agencia EFE. 19 feb 2016. Disponible en: <http:// 
www.efe.com/efe/espana/portada/reino-unido-y-la-ue-logran-unacuerdo-final-dirigido-a-impedir-el-brexit/10010-2844328>

“Tras el Brexit, la EFTA es una opción para el Reino Unido, pero Noruega se opone a su entrada" [en línea]. El Economista.es. 1 jul 2016. Disponible en: <http://www.eleconomista.es/economia/noticias/7677327/07/16/ Tras-el-Brexit-la-EFTA-es-una-opcion-para-el-Reino-Unido-peroNoruega-se-opone.html>

“Tras la victoria del 'BREXIT' Irlanda del Norte y Escocia exigen sendos referéndums para salir de Gran Bretaña” [en línea]. El Periódico Internacional. 24 jun 2016. Disponible en: <http://www.elperiodico. $\mathrm{com} / \mathrm{es} /$ noticias/internacional/escocia-irlanda-del-norte-votan-favorseguir-union-europea-5226085>

ALONSO GARCÍA, Ricardo. Sistema Jurídico de la Unión Europea. 4ª ed. Navarra: Thomson - Civitas, 2014.

ALONSO GARCÍA, Ricardo. Tratado de Amsterdam Versiones consolidadas de los Tratados de la Union Europea y de Funcionamiento de la Unión Europea. Navarra: Civitas - Thomson Reuters, 2012.

ALONSO GARCÍA, Ricardo. Tratado de Lisboa, Tratado. Versiones consolidadas de los Tratados de la Unión Europea y de Funcionamiento de la Unión Europea. $3^{\text {a }}$ ed. s.n: Civitas - Thomson Reuters, s.f.

ALONSO GARCÍA, Ricardo. Tratado de Niza. Versiones consolidadas de los Tratados de la Unión Europea y de Funcionamiento de la Unión Europea. s.n: Edición Civitas - Thomson Reuters, s.f.

MOLINA DEL POZO, Carlos Francisco. Manual de Derecho de laComunidad Europea. $3^{\text {a }}$ ed. Editorial Trivium, Madrid 1997.

ALONSO GARCÍA, Ricardo. Tratados y Legislación Institucional de la Unión Europea. 6a ed. s.n: Edit. Civitas - Thomson Reuters, 2012.

MANGAS, Martín; LIÑÁN NOGUERAS, Diego J. Instituciones y Derecho de la Unión Europea. Madrid: Mc. Graw Hill, 1996.

MOLINA DEL POZO, Carlos Francisco. Evolución histórica y jurídica de los Procesos de Integración en la Unión Europea y en el MERCOSUR. Liber Amicorum Miguel Angel Ciuro Caldani. Buenos Aires: Editorial Eudeba, 2011.

MOLINA DEL POZO, Carlos Francisco. Manual de Derecho de la Comunidad Europea. 3a ed. Madrid: Trivium, 1997.

UNIÓN EUROPEA. Acuerdo sobre el Espacio Económico Europeo - Acta Final - Declaraciones conjuntas - Declaraciones de los Gobiernos de los Estados miembros de las CE y de los Estados de la AELC - Acuerdos Acta aprobada - Declaraciones de una o varias partes contratantes del Acuerdo sobre el Espacio Económico Europeo [en línea]. Disponible en: <http://eur-lex.europa.eu/legal-content/ES/TXT/?uri=CELEX\%3A219 94A0103(01)> 
UNIÓN EUROPEA. Convenio de Schengen (1985) DO L 239, 22.9.2000 [en línea]. Disponible en: <http://eur-lex.europa.eu/collection/eu-law/ treaties-other.html?locale $=\mathrm{es}>$

UNIÓN EUROPEA. Decisión 2006/245/CE del Consejo, de 27 de febrero de 2006, relativa a la celebración, en nombre de la Comunidad Europea y de sus Estados miembros.

UNIÓN EUROPEA. Tratado sobre la Unión Europea - Tratado de Maastricht [en línea]. Disponible en: <http://europa.eu/eu-law/ decision-making/treaties/index_es.htm>

UNIÓN EUROPEA. Tratados Constitutivos de las Comunidades Europeas. Tratados por los que se revisan dichos Tratados. Acta Unica Europea. Oficina de Publicaciones oficiales de las Comunidades Europeas. CECA-CEE-CEEE. Bruselas-Luxemburgo, 1987. 